\title{
Potential Allosteric Sites Captured in Glycolytic Enzymes via Residue-Based Network Models: Phosphofructokinase, Glyceraldehyde-3-Phosphate Dehydrogenase and Pyruvate Kinase
}

\author{
Metehan Celebi ${ }^{1}$, Tuğçe İnan ${ }^{2}$, Ozge Kurkcuoglu ${ }^{2}$, and Ebru Akten ${ }^{1}$ \\ ${ }^{1}$ Kadir Has University \\ ${ }^{2}$ Istanbul Technical University
}

May 30, 2021

\begin{abstract}
Likelihood of new allosteric sites for glycolytic enzymes, phosphofructokinase (PFK), glyceraldehyde-3-phosphate dehydrogenase $(\mathrm{GADPH})$ and pyruvate kinase (PK) was evaluated for bacterial, parasitic and human species. Allosteric effect of a ligand binding at a site was revealed on the basis of low-frequency normal modes via $\mathrm{C} \alpha$-harmonic residue network model. In bacterial PFK, perturbation of the proposed allosteric site outperformed the known allosteric one, producing a high amount of stabilization or reduced dynamics, on all catalytic regions. Another proposed allosteric spot at the dimer interface in parasitic PFK exhibited major stabilization effect on catalytic regions. In parasitic GADPH, the most desired allosteric response was observed upon perturbation of its tunnel region which incorporated key residues for functional regulation. Proposed allosteric site in bacterial PK produced a satisfactory allosteric response on all catalytic regions, whereas in human and parasitic PKs, a partial inhibition was observed. Residue network model based solely on contact topology identified the 'hub residues' with high betweenness tracing plausible allosteric communication pathways between distant functional sites. For both bacterial PFK and PK, proposed sites accommodated hub residues twice as much as the known allosteric one. Tunnel region in parasitic GADPH with the strongest allosteric effect among species, incorporated the highest number of hub residues. These results clearly suggest oneto-one correspondence between the degree of allosteric effect and the number of hub residues in that perturbation site, which increases the likelihood of its allosteric nature.
\end{abstract}

\section{Hosted file}

Manuscript_Celebi_Inan_Kurkcuoglu_Akten_May2021.docx available at https://authorea. com/users/416750/articles/524166-potential-allosteric-sites-captured-in-glycolyticenzymes-via-residue-based-network-models-phosphofructokinase-glyceraldehyde-3-phosphatedehydrogenase-and-pyruvate-kinase 